\title{
Research on Multi-attribute Vertical Handoff Algorithm Based on Motion Trend Quantification in Internet of Vehicle
}

\author{
Mingji Yang ${ }^{1, a}$, Ye $\mathrm{Wu}^{1, b}$ and Rong $\mathrm{Cui}^{2, \mathrm{c}}$ \\ ${ }^{1}$ Harbin University of Science and Technology \\ Harbin, China, 150080 \\ ${ }^{2}$ No. 703 Research Institute of China Shipbuilding Industry Corporation \\ Harbin, China, 150078 \\ a18845646347@163.com, buye930825@163.com, ccuirong921@163.com
}

Keywords: Internet of Vehicle; wireless heterogeneous network; motion trend; multiple attribute decision; vertical handoff.

\begin{abstract}
Due to the ambiguity of vehicle terminal motion trends, base stations can easily initiate handover blindly and result in higher handover failure rate. An optimized algorithm is proposed to optimize existing vertical handoff algorithm in the LTE - WiMAX - WAVE heterogeneous wireless network system. The proposed algorithm uses the motion trend quantification to estimate the target area and restrict unnecessary handoff so as to increase success rate of handoff. Computer simulation results on fading channels show that the proposed algorithm can reduce the handover failure rate in handover, thus improving the handover performance of the network and ensuring the network update timely.
\end{abstract}

\section{INTRODUCTION}

At present, many researchers have achieved good results on the vertical handoff between heterogeneous wireless networks. Yang et $\mathrm{al}^{[2,3,4]}$ proposed a algorithm which is based on fuzzy logic and neural network, the algorithm quantifies some factors which are difficult to quantify and improves the accuracy of decision., However, this algorithm has high complexity and difficult to implement. Ma et al ${ }^{[5,6]}$ proposed a predictive vertical handoff algorithm, which allows the user to know the change of the network parameters in advance, and thus to switch effectively. The disadvantage of this algorithm is that when the user terminal moves to a new network coverage area, the efficiency of the algorithm decreases or even loses due to the lack of historical movement information.

\section{MOBILE TREND JUDGMENT AND QUANTIFICATION}

By the Doppler shift formula, we can get $v_{m} \cos \theta=\left(f_{c}-f_{r}\right) / f_{c} v_{c}$, Here the $f_{c}$ is the frequency of the transmitted signal, $f_{r}$ is the received signal frequency, $\left|f_{c}-f_{r}\right|$ is Doppler shift $f_{d}$, so:

$$
\left|v_{m} \cos \theta\right|=\left(f_{d} / f_{c}\right) v_{c} .
$$

According to formula (1), we can calculate component $\left|v_{m} \cos \theta\right|$ of the vehicle terminal speed in the direction of the target base station. And the motion trend parameters $\left|v_{m} \cos \theta\right|$ are obtained by the velocity components.

\subsection{Calculate the maximum Doppler shift}

According to the proposed Doppler shift estimation algorithm ${ }^{[7,8,9]}$, the algorithm is based on the cyclic prefix autocorrelation function. Therefore, Doppler shift can be obtained by calculating the 
autocorrelation function of the symbol. To eliminate the effect of inter-carrier interference on the calculation, a guard interval can be inserted between consecutive symbols. As shown in Figure 1. Then a complete OFDM symbol length $T_{s}$ is $T_{s}=(G+N) T$. T Is the sampling interval, and $G$ is the guard interval signal number.

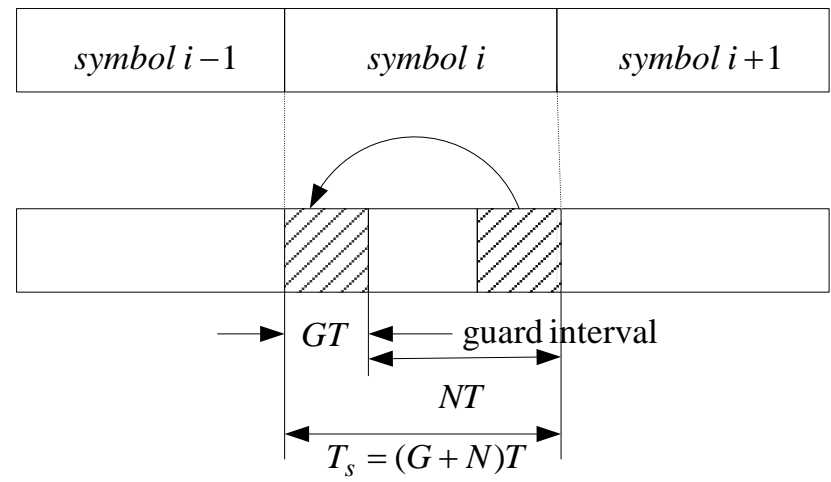

Figure 1 the loop structure of symbol OFDM

The discrete form of the received signal is:

$$
\begin{aligned}
r(m)= & s(m)+n(m)=r_{i}(m)+j r_{q}(m) \\
& =\left[s_{i}(m)+n_{i}(m)+j\left[s_{r}(m)+n_{r}(m)\right] .\right.
\end{aligned}
$$

In the equation (2), the transmitted signal is $s(m)$, the noise signal is $n(m)$, and the real part of the received signal is $r_{i}(m)$, the imaginary part of the received signal is $r_{q}(m)$. We can calculate the Expected value of $s(m)$ and $n(m)$ :

$$
\begin{aligned}
& E\left[s_{i}{ }^{2}(m)\right]=E\left[S_{q}{ }^{2}(m)\right]=\frac{E\left[s^{2}(m)\right]}{2}=\frac{\sigma^{2}}{2} . \\
& E\left[n_{i}{ }^{2}(m)\right]=E\left[n_{q}{ }^{2}(m)\right]=\frac{N_{0}{ }^{2}}{2} .
\end{aligned}
$$

Considering the influence of time-selective fading on the channel caused by $f_{d}$, the autocorrelation function of the received signal is

$$
C_{r_{i} r_{i}}(\tau)=C_{r_{q} r_{q}}(\tau)=E\left[r_{i}(m) r_{i}(m+\tau)\right]=\frac{\sigma^{2} J_{0}\left(2 \pi f_{d} \tau\right)}{2}+\frac{N_{0} \delta(\tau)}{2} .
$$

Which $J_{0}(\bullet)$ is the first order zero-order Bessel function;

After the guard interval is inserted, the autocorrelation of the symbols OFDM is obtained:

$$
\begin{aligned}
c\left(f_{d}, N T\right)= & \frac{\sum_{m=1}^{G} r_{i}\left(i^{\prime}+(m+N) T\right) r_{i}\left(i^{\prime}+m T\right)}{2 \sum_{k=1}^{G} r_{i}{ }^{2}\left(i^{\prime}+(m+N) T\right)} \\
& +\frac{\sum_{m=1}^{G} r_{q}\left(i^{\prime}+(m+N) T\right) r_{q}\left(i^{\prime}+m T\right)}{2 \sum_{k=1}^{G} r_{q}{ }^{2}\left(i^{\prime}+(m+N) T\right)} .
\end{aligned}
$$

Among them $i^{\prime}=(i-1)(N+G) T$. And $s(m)$ and $n(m)$ independent of each other, in the case of sufficient samples, then 


$$
c\left(f_{d}, N T\right) \approx \frac{C_{r_{1} r_{1}}(N T)}{N_{0}+\sigma^{2}}+\frac{C_{r_{q} r_{q}}(N T)}{N_{0}+\sigma^{2}} .
$$

Put formula (5) into formula (7) was:

$$
c\left(f_{d}, N T\right) \approx \frac{1}{1+N_{0} / \sigma^{2}} J_{0}\left(2 \pi f_{d} N T\right)+\frac{1}{1+\sigma^{2} / N_{0}} .
$$

Then on both sides of the formula (8) for the autocorrelation operation, you can get the maximum Doppler frequency shift $f_{d}$.

\subsection{The judgment of terminal motion state and the calculation of the velocity component}

In this paper, we use the difference of the two RSS to judge the motion state of the vehicle terminal. A set of RSS samples for a period of time is $\left\{r_{1}, r_{2}, \cdots r_{N}\right\}$, and $N=M L$, Sampling values in the set are grouped into groups of L to obtain a total of M samples. After sampling, a value of $r_{i, \max }$, minimally disturbed by shadow fading, is selected in the sample set as a reference value to facilitate comparison with other values. $\Delta r=r_{i, \max }-r_{i-1, \max }$ as a parameter to judge the motion state of the terminal.

There are two cases: (1) $\Delta r>0$ : Indicates that the terminal is moving towards the target base station. (2) $\Delta r<0$ : Indicates that the terminal is moving away from the target base station.

From equation (1), $\left|v_{m} \cos \theta\right|$ is the component of terminal moving speed in the direction of each target base station. Take

$$
v=v_{m} \cos \theta=\left\{\begin{array}{ll}
+\left|v_{m} \cos \theta\right|, & \Delta_{r}>0 \\
-\left|v_{m} \cos \theta\right|, & \Delta_{r}<0
\end{array} .\right.
$$

From the above equation, when $v$ is positive, it indicates that the current vehicle terminal is moving toward the coverage area of the target base station, the value is large, the trend is more obvious. Otherwise, it indicates that the terminal is moving away from the target base station coverage.

\subsection{Generate moving trend intensity parameters and motion trajectory analysis}

In this paper, the trend of terminal motion is one of the determinants, so we need to normalize the moving trend of the terminal to deal with the contrast of other parameters. So we design parameters as follow:

$$
T=\frac{\arctan [v(x) / \alpha]}{\pi}+\frac{1}{2} .
$$

When the terminal is moving back and forth between the two base stations, it may trigger frequent network switching, which has a great impact on the service of the terminal. In this case, the trajectory of the terminal has a round-trip characteristic. In the previous calculation of the basis of speed component information, we introduce the following formula to determine the trajectory of the terminal

$$
\left|\sum_{n=i-M}^{i} v_{n}\right| \leq \varepsilon
$$

When the sum of the absolute values of the components of the terminal in each base station direction is calculated, and when the calculated result is not greater than the set judgment value $\varepsilon$, the movement tendency is considered to be round-trip, limit the number of switching to ensure the 
continuity of the terminal service. While reducing the number of switching, the algorithm efficiency has been improved accordingly.

\section{VERTICAL MOVEMENT ALGORITHM BASED ON MOTION TREND}

The switching strategies proposed in this paper include normalization parameters, weighting factors, generating multiple attribute decision values, and decision switching.

\subsection{Normalized parameters}

In order to make the network attribute parameters in the heterogeneous network comparability, we should normalize the parameters of each network.

(1) The normalization function of RSS: $R(x)$ is defined as the received signal strength of each target network, $R_{t h}(x)$ is the minimum received signal strength value that satisfies the current service, $R_{\max }(x)$ is the maximum received signal strength of the target network. $x$ Indicates the current position of the vehicle terminal coordinates. The normalization of RSS is as follows:

$$
\mu_{R}=\left\{\begin{array}{ll}
0, & 0 \leq R(x)<R_{t h} \\
\frac{R(x)-R_{t h}(x)}{R_{\max }-R_{t h}(x)}, & R(x) \geq R_{t h}(x)
\end{array} .\right.
$$

(2) The normalization function of BW: $B(x)$ is defined as the available bandwidth of each target network, $B_{\max }(x)$ is the maximum value of the available bandwidth. The normalization of $\mathrm{BW}$ is as follows:

$$
\mu_{B W}=\left\{\begin{array}{ll}
\frac{B(x)}{B_{\max }(x)}, & 0 \leq B(x)<B_{\max } \\
1, & R(x) \geq R_{t h}(x)
\end{array} .\right.
$$

In the above analysis, the motion trend parameter $T$ has been normalized, here take $\mu_{T}=T$.

\subsection{Compute weighting factors and generate multiple attribute decision values}

The design weighting factor is as follows:

$$
w_{i}=\frac{\sigma_{i}}{\sigma_{R}+\sigma_{B T}+\sigma_{T}} .
$$

From the formula (16), we can see that the weighting factor will change when the parameters of the network are changed. It is obvious that some parameters have obvious change in the judgment.

$\sigma_{i}$ Is the standard deviation of the normalized variables $\mu_{i, 1}(x), \mu_{i, 2}(x), \cdots \mu_{i, n}(x)$ of the candidate base stations, Where $i$ is $R, B W$ or $T, R, B W$, respectively, s represent three network attribute parameters. The second subscript represents the target network, so

$$
\sigma_{i}=\sqrt{\frac{1}{n} \sum_{k=1}^{n}\left[\mu_{i, k(x)}-\frac{1}{n} \sum_{k=1}^{n} \mu_{i, k}\right]^{2}} .
$$

After obtaining the weighting factor of each network attribute, the multi-attribute decision value can be obtained. Using this value can evaluate the performance of each target network, so the multi-attribute decision value is

$$
D_{k}(x)=w_{R} \mu_{R, k(x)}+w_{B W} \mu_{B W, k(x)}+w_{T} \mu_{T, k(x)} .
$$


$k$ is used to identify different networks.

\subsection{Switching decisions}

After calculating the multiple attribute decision value of each alternative base station, the handover decision can be implemented. First of all, to determine the formula (12), that is to analyze whether the vehicle terminal to round-trip movement, if the condition of velocity component satisfies (12), the decision is not made to reduce over-switching and algorithm data processing capacity; otherwise, the target network with the largest multi-attribute decision value is determined as the optimal network, that is:

$$
D_{b}(x)=\max \left\{D_{1}(x), D_{1}(x), \cdots D_{n}(x)\right\} .
$$

From formula (19), $D_{b}(x)$ corresponds to the best network performance. Then the decision strategy is: when the terminal has access to the corresponding network $D_{b}(x)$ before the decision, then we does not need to switch; otherwise, switch to the corresponding network $D_{b}(x)$.

\section{Simulation analyses}

\subsection{Simulation Experimental Scene}

The heterogeneous network model of this algorithm is composed of WAVE, LTE and WiMAX. Simulation scenarios shown in Figure 2 , the position coordinates of point $A$ are $(15,190)$, the position coordinate of point $B$ is $(200,190)$, the position coordinate of point $C$ is $(200,0)$, and the distance between point $A$ and point $B$ is $15 \mathrm{~km}$, The distance from point $B$ to the point $C$ is $15 \mathrm{~km}$, and the base stations WiMAX 1, WiMAX 2, WiMAX 3, WiMAX 4 corresponding to coordinates $(105,240),(200$, 190), (235, 120) and $(175,105)$. The base stations LTE 1, LTE 2, LTE 3 corresponding to coordinates $(60,160),(210,160),(135,30)$. Network simulation parameters as shown in Table 1:

Table 1 Network parameters

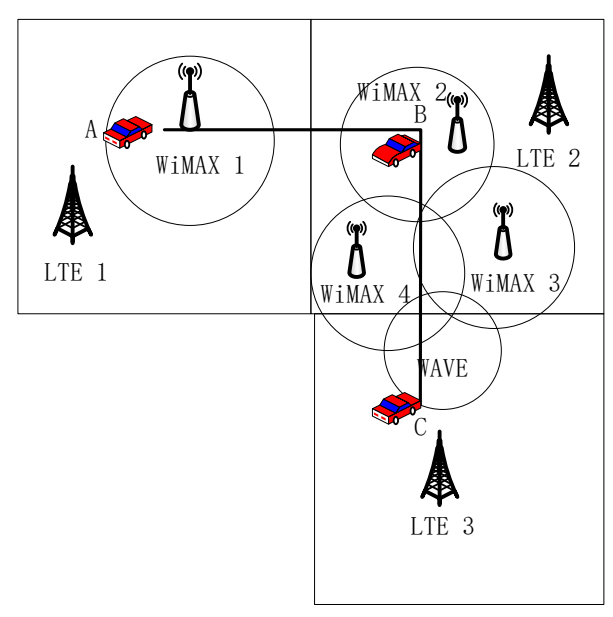

\begin{tabular}{lccc}
\hline parameters & LTE & WiMAX & wAVE \\
\hline $\begin{array}{c}\text { Transmission power } \\
(\mathrm{dBm})\end{array}$ & 44 & 30 & 27 \\
$\begin{array}{c}\text { Coverage }(\mathrm{km}) \\
\begin{array}{c}\text { Access bandwidth } \\
(\text { Mbit/s) }\end{array}\end{array}$ & Full coverage & 10 & 1 \\
\hline
\end{tabular}

Figure 2 Simulation scenario

\subsection{Simulation results}

Compared with the handover algorithm which is not quantized in the literature ${ }^{[10]}$ (MHA), the terminal needs to switch over the network due to the area covered by different target networks during the process of the movement. The handover process has a certain delay. If the vehicle terminal is not within the above range, the handover is considered to fail. Figure 3 shows the trend of the failure rate of the two handover algorithms. Although the handover failure rates of the two algorithms increase 
with the increase of the vehicle terminal speed, considering the unnecessary handover due to the round-trip motion, in this paper, we analyze the movement trajectory of the terminal and analyze the moving trend of the vehicle accurately, which reduces the failure rate of the switch. The graph shows that the rate of rising of the curve is slow. Compared with the algorithm, MHA failure rate is higher, as shown in the graph; the MHA curve shows a rapid rise.

Figure 4 compares the data rate of the terminal with the speed of the terminal moving under the two switching algorithms. When the terminal switch fails, the data rate drops to zero, which will seriously affect the quality of service on the terminal. With the increase of the rate, it can be found that the user data rate of the MHA is significantly reduced, which is mainly due to the switch failure caused by the unreasonable handover decision, resulting in loss of connection. Because the coverage area of WLAN is small, the handover failure occurs when the user switches to the WLAN network. Therefore, users can not make full use of the high-speed transmission of WLAN, which is another reason for the decrease of MHA data rate.

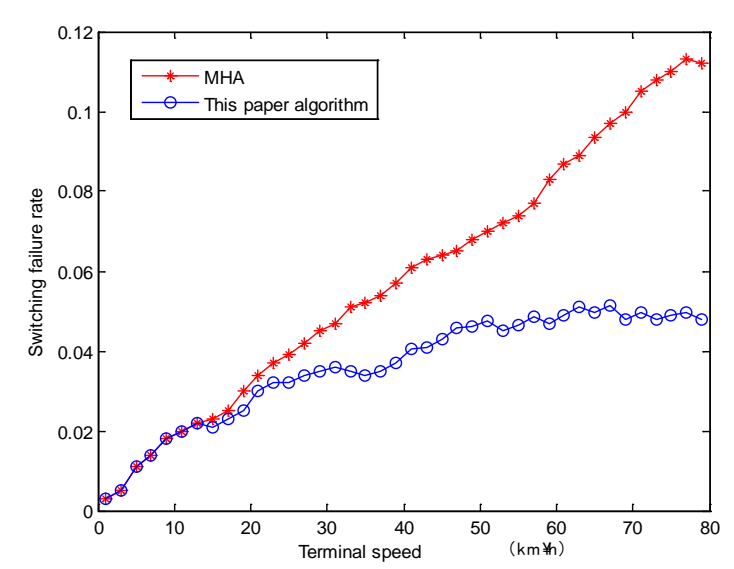

Figure 3 switch failure rate comparison

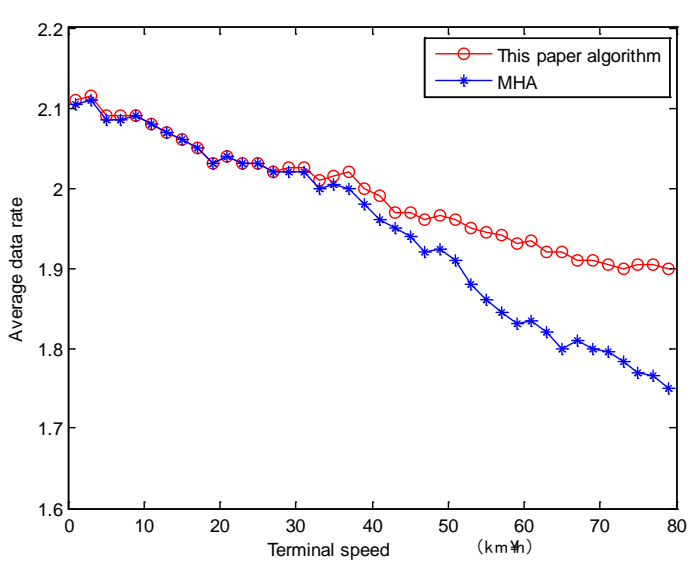

Figure 4 terminal data rate comparison

\section{Conclusions}

This paper presents a multi-attribute vertical handoff algorithm which quantifies the moving trend of the terminal. Compared with the traditional multi-attribute vertical handoff algorithm, the network parameters are quantized in this paper, and the terminal velocity can be quantified at the same time. Analysis of the trajectory of the terminal, effectively reducing the number of switching to ensure the continuity of the terminal service, but also improve the user data rate.

\section{References}

[1] AHMED A, BOULAHIA L M, and GAITI D. Enabling vertical handover decisions in heterogeneous wireless networks: A state-of-the-art and a classification [J]. IEEE Communications Surveys \& Tutorials, 2014, 16(2): 776-811.

[2] A. Calhan, C.Çeken. An adaptive neuro-fuzzy based vertical handoff decision algorithm for wireless heterogeneous networks[C].Personal Indoor and Mobile Radio Communications (PIMRC), 2010: 2271-2276.

[3] YANG T and RONG P. A fuzzy logic vertical handoff algorithm with motion trend decision[C]. 2011 6th IEEE International Forum on Strategic Technology (IFOST), Harbin, China, 2011: 1280-1283. 
[4] STEVENS-NAVARRO E, LIN Y, WONG V W S. An MDP-based vertical handoff decision algorithm for heterogeneous wireless networks [J]. IEEE Transactions on Vehicular Technology, 2008, 57(2): 1243-1254.

[5] LI Bin and LIU Shengmei. Vertical handoff algorithm based on mobility prediction [J]. Communication and Network, 2013, 39(1): 93-95.

[6] B.J. Chang, J.F. Chen, C.H. Hsieh, et al. Markov decision process-based adaptive vertical handoff with RSS prediction in heterogeneous wireless networks[C].Wireless Communications and Networking Conference, 2009. WCNC 2009, 2009: 1-6.

[7] MENG J and CHEN J. Doppler spread estimation for mobile OFDM systems [J]. Electronic Science and Technology, 2011, 24(6): 1-3.

[8] Schober H, Jondrale. Velocity estimation for OFDM based communication systems. IEEE Vehicular Technology Cont[C]. 2002. 715-718.

[9] Lin Hao and Siohan P. Robust channel estimation for OFDM/OQAM [J]. IEEE Communication Letters, 2009, 13(10): 724-726.

[10] WANG S,FAN C,HSUC,et al. A vertical handoff method via self-selection decision tree for internet of vehicles [J].IEEE Systems Journal, 2014, 30(10):443-458. 